\title{
A Low-Frequency Model for E-Field and B-Field Coupling into a Folded Antenna with Two Gaps
}

M. P. Perkins, M. M. Ong, C. L. Robbins

January 23, 2012

2012 IEEE Symposium on Electromagnetic Compatibility Pittsburgh, PA, United States

August 5, 2012 through August 10, 2012 
This document was prepared as an account of work sponsored by an agency of the United States government. Neither the United States government nor Lawrence Livermore National Security, LLC, nor any of their employees makes any warranty, expressed or implied, or assumes any legal liability or responsibility for the accuracy, completeness, or usefulness of any information, apparatus, product, or process disclosed, or represents that its use would not infringe privately owned rights. Reference herein to any specific commercial product, process, or service by trade name, trademark, manufacturer, or otherwise does not necessarily constitute or imply its endorsement, recommendation, or favoring by the United States government or Lawrence Livermore National Security, LLC. The views and opinions of authors expressed herein do not necessarily state or reflect those of the United States government or Lawrence Livermore National Security, LLC, and shall not be used for advertising or product endorsement purposes. 


\title{
A Low-Frequency Model for E-Field and B-Field Coupling into a Folded Antenna with Two Gaps
}

\author{
Michael P. Perkins, Mike M. Ong, Christopher L. Robbins \\ Lawrence Livermore National Laboratory \\ 7000 East Avenue, Livermore, CA 94550, USA \\ perkins22@llnl.gov
}

\begin{abstract}
Electric field coupling into electrically small monopoles/dipoles and magnetic field coupling into electrically small loop antennas has been investigated extensively due to their applicability to a wide range of applications. However, under certain conditions electrically small folded antenna structures exist in which both coupling mechanisms must be included simultaneously in order to perform an accurate system analysis. In this paper we present a low frequency model that includes both electric and magnetic field coupling simultaneously for a folded antenna with two gaps. Values for a circuit model are found using an electrostatic finite element code and a full wave frequency domain finite element code. The circuit model is then validated by a full wave finite difference time domain code. For the time domain analysis the antenna structure is excited by fields from a lightning pulse. The time domain simulation has excellent agreement with the circuit model that is presented.
\end{abstract}

\section{INTRODUCTION}

Coupling of unwanted electric fields (E-fields) and magnetic fields (B-fields) into systems can often harm systems and components. In order to verify that a system is immune, analysis of different coupling scenarios must be performed. Cables such as power cords can extend from devices in many different ways. When performing a coupling analysis for low frequency pulses good results can often be obtained by accounting for only E-field coupling into monopole type antenna configurations or only B-field coupling into loop type antenna configurations that are formed by the cable. When the cable forms a folded antenna with a gap at each end of the cable, both E-field and B-field coupling can occur simultaneously. Often one can neglect the B-field coupling when the ratio of $\mathrm{E} / \mathrm{B}$ produced by the pulse is large enough and the gap capacitances are small. However, if the system is in a cavity type of structure where E/B is decreased, one cannot neglect the B-field coupling. This coupling scenario is one that has received little discussion.

One important application for this coupling scenario is for electro-explosive device safety [1]-[3]. Many times electroexplosive devices are stored in bunker type facilities. The rebar in the structure serves as a lightning protection system. However, penetrations and discontinuities in the rebar can cause E-fields and B-fields to be created inside the facility when the facility is struck by lightning [1]. A cable that is attached to the detonator can behave as an antenna and cause a voltage to develop over the detonator [2], [3]. If the voltage is too large an electrical spark can occur that may ignite the explosives in the detonator [2], [3]. Thus, it is important for worker safety and to protect property to make sure that such coupling does not cause an unintended explosion.

The coupling into the cable we will investigate is closely related to folded dipoles [4]-[7] and loaded loops [8]-[10]. In analysing a folded antenna it is often convenient to decompose the voltages on the antenna into two modes, a Tline mode (differential mode), and a dipole mode (common mode). One must take care when analysing coupling into folded antenna structures that the appropriate modes and excitations are taken into account [11]-[13]. When coupling into a folded antenna with two gaps we will see that the differential mode is excited by a loop antenna type coupling and the common mode by a monopole type coupling. The distributed capacitance of the structure and the capacitances formed at the gaps will determine the total voltages developed over each gap.

To help illustrate the coupling we will analyse the two folded antennas shown in Fig. 1. All dimensions have been set equal to multiples of $2 \mathrm{~mm}$ to aid FDTD simulations. The wire cross-section is square with sides $2 \mathrm{~mm}$. Both antennas are connected to a T-line/load capacitance (red in Fig. 1) which extends into the ground plane through a small aperture. In Fig. 1 (a), the second gap is formed at the end of the cable and represented by a capacitance below the ground plane on the bottom right side of the figure. In Fig. 1 (b), the second gap is between two cables. For each structure we will refer to the two gaps as the load gap and the parasitic gap. The ground plane is shown on the bottom of the figure, not shown is the top conductor at $0.8 \mathrm{~m}$ which forms a parallel plate waveguide with the bottom plane. In Sect. II we will create a circuit model for each of these configurations. This circuit model is validated using an FDTD analysis in Sect. III.

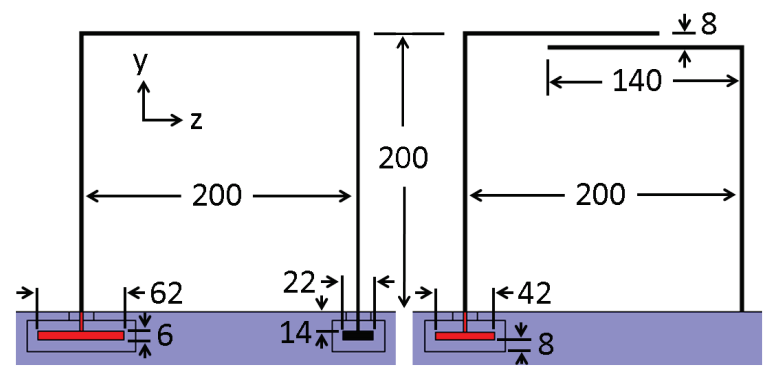

(a)

(b)

Fig. 1. Folded antenna structures with dimensions in millimeters and two gaps. The red portion indicates the load. Figure 1(a) will be referred to as the continuous case and Fig. 1 (b) will be referred to as the discontinuous case. 


\section{CIRCUIT MODEL}

The proposed low frequency lumped element circuits for the E-field and B-field coupling are shown in Fig. 2. The circuit in Fig. 2 (a) is for E-field coupling and the circuit in Fig. 2 (b) is for B-field coupling. In these circuits $C_{L}$ represents the T-line/load capacitance (for remainder of paper referred to as load capacitance). In Fig. 2 (a), $v_{S E}$ is the source voltage excited by E-field coupling developed at the load end of the antenna with no load is attached. It is related to the effective height of the antenna by $\mathrm{v}_{\mathrm{SE}}=\mathrm{h}_{\mathrm{eff}} \cdot \mathrm{E}$. The antenna capacitance for E-field coupling is $C_{A E}$. In Fig. 2 (b), $v_{S B}$ is the source voltage excited by $B$-field coupling and is given by $v_{S B}$ $=\partial / \partial \mathrm{t} \iint \mathrm{B} \cdot \mathrm{ds}$, where $\mathrm{ds}$ is the incremental surface of the loop formed by the folded antenna. $C_{P B}$ and $C_{A B}$ represent distributed capacitances of the structure that have been lumped such that $C_{P B}$ is attributed to the capacitance at the parasitic end of the cable and $C_{A B}$ is attributed to the load end.

The circuit in Fig. 2 (a) is the same as that of a small monopole or dipole with a load attached. We see that Fig. 2 (b) is different than that of an ordinary small loop antenna. A conventional small loop antenna has the same circuit topology as Fig. 2 (a) with $\mathrm{v}_{\mathrm{SE}}$ replaced by $\mathrm{v}_{\mathrm{SB}}$ and $\mathrm{C}_{\mathrm{AE}}$ replaced by the inductance of the loop antenna. In Fig. 2 (b) we see that the low impedance from the inductance has been neglected in comparison to the two capacitances $C_{P B}$ and $C_{A B}$. The voltages developed over $C_{L}$ for the E-field coupling circuit, $v_{L E}$, and the B-field coupling circuits, $\mathrm{V}_{\mathrm{LB}}$, are given by (1) and (2), and the voltage developed over $\mathrm{C}_{\mathrm{PB}}$ is given by (3).

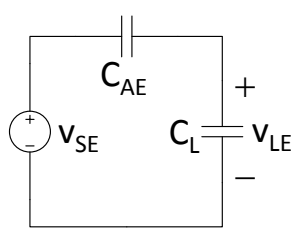

(a)

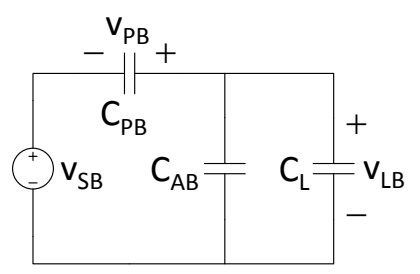

(b)
Fig. 2. Circuits for (a) E-field coupling and (b) B-field coupling into folded antenna structure with multiple gaps.

$v_{L E}=v_{S E} \frac{C_{A E}}{C_{A E}+C_{L}}=E \cdot h_{\text {eff }} \frac{C_{A E}}{C_{A E}+C_{L}}$

$v_{L B}=v_{S B} \frac{C_{P B}}{C_{P B}+C_{A B}+C_{L}}=\frac{\partial}{\partial t} \iint B \cdot d s \frac{C_{P B}}{C_{P B}+C_{A B}+C_{L}}$

$v_{P B}=-v_{S B} \frac{C_{A B}+C_{L}}{C_{P B}+C_{A B}+C_{L}}=-\frac{\partial}{\partial t} \iint B \cdot d s \frac{C_{A B}+C_{L}}{C_{P B}+C_{A B}+C_{L}}$

We will now begin to determine the circuit elements for the two configurations shown in Fig. 1. The first circuit elements that we will determine are the capacitances for the E-field coupling circuit. Three different loads will be used. The loads are all square with cross sectional lengths of 22,42 , and $62 \mathrm{~mm}$. The capacitances are computed using the electrostatic finite element code MAXWELL $3 \mathrm{D}$ by placing the loads at 1 $\mathrm{V}$, and the ground plane at $0 \mathrm{~V}$. The capacitances are determined using the relation $\mathrm{C}=2 \mathrm{~W}_{\mathrm{e}} / \mathrm{V}^{2}$, where $\mathrm{W}_{\mathrm{e}}$ is the electrostatic energy. The electrostatic energy densities used to compute the capacitances are shown in Fig. 3 and the computed capacitance values are given in Table I.

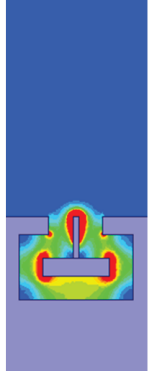

(a)

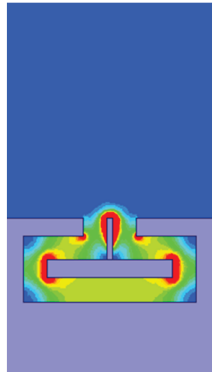

(b)

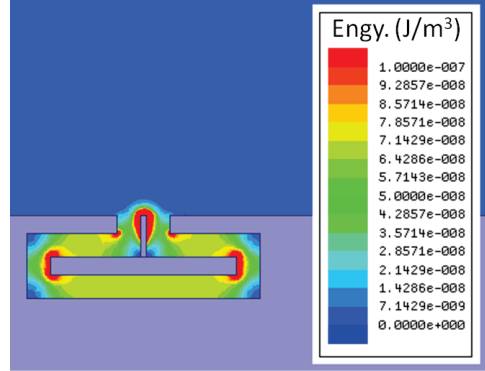

(c)
Fig. 3. Energy density used in computing the capacitances for loads with cross sectional lengths of (a) $22 \mathrm{~mm}$, (b) $42 \mathrm{~mm}$, and (c) $62 \mathrm{~mm}$.

TABLE I

LOAD CAPACITANCES IN PICOFARADS

\begin{tabular}{|c|c|c|}
\hline $\mathbf{C}_{\mathrm{L} 1}$ & $\mathbf{C}_{\mathrm{L} 2}$ & $\mathbf{C}_{\mathrm{L3}}$ \\
\hline 2.84 & 6.98 & 12.90 \\
\hline
\end{tabular}

Next, we compute $\mathrm{C}_{\mathrm{AE}}$, once again using the relationship between electrostatic energy and capacitance. This is accomplished by removing the load so that the antenna is open circuited and placing $1 \mathrm{~V}$ on the antenna. The resultant energy density is shown in Fig. 4 (a) for the continuous folded antenna case and results are given in the second column of Table II. One can perform a check on the calculations by attaching the load to the antenna and computing the total capacitance, as illustrated in Fig. 4 (b) for discontinuous folded antenna case. The results are given in the last three columns of Table II. By adding the load capacitance values given in Table $I$ to the values for $\mathrm{C}_{\mathrm{AE}}$, we see that excellent agreement is found with the last three columns of Table II.

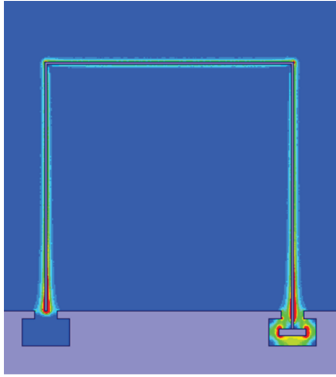

(a)

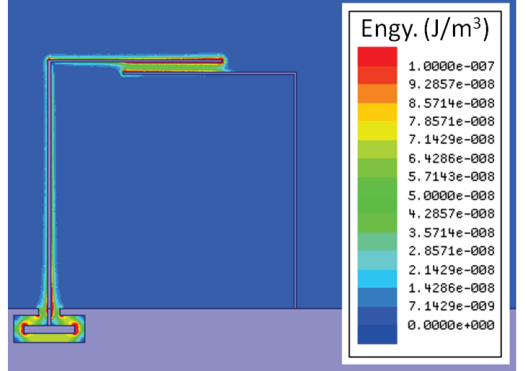

(b)
Fig. 4. Energy density used to compute (a) $\mathrm{C}_{\mathrm{AE}}$ for the continuous case and (b) $\mathrm{C}_{\mathrm{AE}}+\mathrm{C}_{\mathrm{L} 3}$ for the discontinuous case.

TABLE II

CAPACITANCES FOR ANTENNAS WITH DIFFERENT LOADS IN PICOFARADS

\begin{tabular}{|l|c|c|c|c|}
\hline & Open $\left(\mathbf{C}_{\mathbf{A E}}\right)$ & $\mathbf{C}_{\mathrm{AE}}+\mathbf{C}_{\mathbf{L 1}}$ & $\mathbf{C}_{\mathrm{AE}}+\mathbf{C}_{\mathbf{L} 2}$ & $\mathbf{C}_{\mathrm{AE}}+\mathbf{C}_{\mathbf{L 3}}$ \\
\hline Continuous & 9.60 & 12.30 & 16.44 & 22.36 \\
\hline Discontinuous & 4.64 & 7.34 & 11.48 & 17.40 \\
\hline
\end{tabular}


The frequency domain full wave finite element solver HFSS is used to determine the remaining circuit elements in Fig. 2. A uniform plane wave at $1 \mathrm{MHz}$ with $\mathrm{E}=1 \mathrm{~V} / \mathrm{m}$ excites the structure $\left(\mathrm{e}^{+\mathrm{j} \omega \mathrm{t}}\right.$ time dependence). The E-field is polarized in the $\mathrm{y}$-direction and propagates in the z-direction, such that the B-field is normal to the loop formed by the folded antenna. Line integrals of the E-field are than performed from ground to the antenna at both gaps to determine the voltages at each gap. When the load is removed (open circuit), the real part of the voltage gives $\mathrm{v}_{\mathrm{SE}}$. This is illustrated in Fig. 5 (a) for the continuous case showing the resultant magnitude for the real part of the E-field. This voltage is the same for both gaps. By attaching a load to the antenna, as illustrated in Fig. 5 (b) for the discontinuous case and using (1), one can verify the circuit for E-field coupling. The results are given by the second column in Table III. The real voltage developed is independent of the direction of the $\mathrm{B}$-field relative to the loop normal.

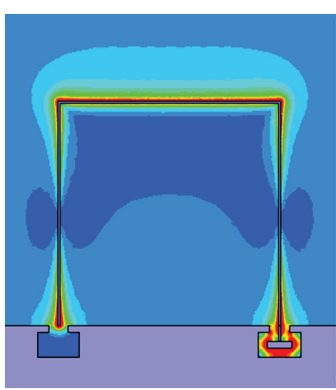

(a)

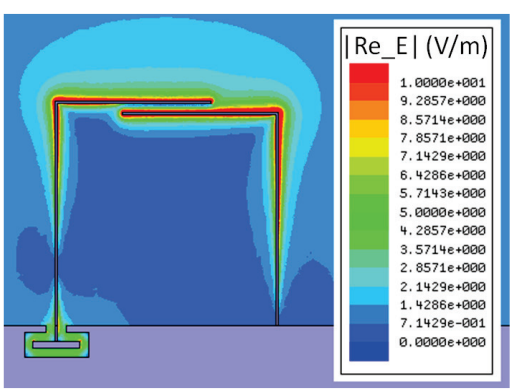

(b)
Fig. 5. Magnitude of the real E-field developed over antennas for (a) the continuous case with no load, and (b) the discontinuous case with $\mathrm{C}_{\mathrm{L} 3}$.

To find $\mathrm{v}_{\mathrm{SB}}$, one simply adds the magnitude of the imaginary voltages developed at the load and parasitic gap. The magnitude of the imaginary E-field developed over the antennas is illustrated in Fig. 6. The results are given by the last two columns of Table III. Note that the imaginary voltage developed at the parasitic gap is $180^{\circ}$ out of phase with the one at the load gap and approximately equal in magnitude only when $C_{P B}$ equals $C_{A B}+C_{L}$.

The remaining capacitances, $C_{P B}$ and $C_{A B}$, were found by two different methods. In the first method, ratios of (2) and (3) were used for cases with two different loads. This system of equations could then be solved for $C_{P B}$ and $C_{A B}$. The results were most accurate when the open circuit case was not used, presumably due to small effects from the fringe fields. These results indicated that $C_{P B}+C_{A B}=C_{A E}$. This allows one to substitute $C_{A E}$ into the denominators of (2) and (3), which could then be solved for $\mathrm{C}_{\mathrm{PB}}$ and $\mathrm{C}_{\mathrm{AB}}$, as given by (4) and (5).

Using values in Table III along with (4) and (5) we calculate that for the continuous case $\mathrm{C}_{\mathrm{PB}}=6.19 \mathrm{pF}, \mathrm{C}_{\mathrm{AB}}=3.41$ $\mathrm{pF}$, and for the discontinuous case $\mathrm{C}_{\mathrm{PB}}=2.17 \mathrm{pF}, \mathrm{C}_{\mathrm{AB}}=2.47$ $\mathrm{pF}$. By comparing the real voltages to the imaginary voltages in Table III, we see that the E-field coupling develops voltages that are much larger than the imaginary voltages for the given load capacitances. If $\mathrm{E} / \mathrm{B}$ were no longer the speed of light, as in a shorted cavity, this will no longer be the case.

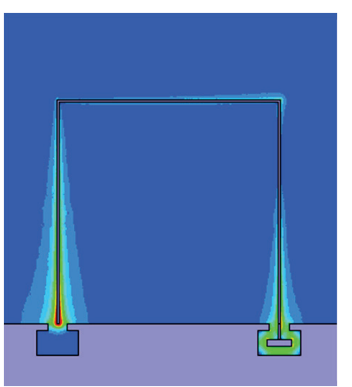

(a)

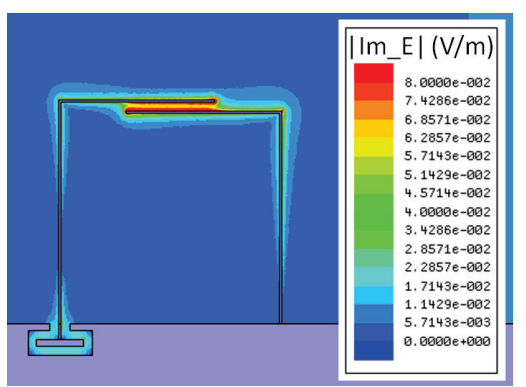

(b)
Fig. 6. Magnitude of imaginary E-field developed over antennas for (a) the continuous case with no load, and (b) the discontinuous case with $\mathrm{C}_{\mathrm{L} 3}$.

TABLE III

CALCUlated LoAd and PaRasitic Gap Voltages in Millivolts

\begin{tabular}{|l|c|c|c|}
\hline & Re_v & $\mathbf{I m}_{-} \mathbf{v}\left(\mathbf{v}_{\mathbf{L B}}\right)$ & $\mathbf{I m}_{-} \mathbf{v}\left(\mathbf{v}_{\mathbf{P B}}\right)$ \\
\hline Continuous, Open & $-85.40\left(\mathbf{v}_{\mathbf{S E}}\right)$ & -0.5380 & 0.3034 \\
\hline Continuous, CL1 & -66.60 & -0.4201 & 0.4220 \\
\hline Continuous, CL2 & -49.84 & -0.3149 & 0.5272 \\
\hline Continuous, CL3 & -36.62 & -0.2319 & 0.6105 \\
\hline Discontinuous, Open & $-95.60\left(\mathbf{v}_{\mathbf{S E}}\right)$ & -0.3790 & 0.4420 \\
\hline Discontinuous, CL1 & -60.43 & -0.2394 & 0.5818 \\
\hline Discontinuous, CL2 & -38.64 & -0.1530 & 0.6682 \\
\hline Discontinuous, CL3 & -25.48 & -0.1008 & 0.7286 \\
\hline
\end{tabular}

$C_{P B}=\left|\frac{v_{L B}}{v_{S B}}\right|\left(C_{A E}+C_{L}\right)$

$C_{A B}=\left|\frac{v_{P B}}{v_{S B}}\right|\left(C_{A E}+C_{L}\right)-C_{L}$

\section{CIRCUIT VALIDATION USING FDTD}

To test the model that was developed we will use the FDTD-PIC code VORPAL to perform a full wave analysis in the time domain. We will simulate both antennas shown in Fig. 1 with various loads for a sinusoidal excitation (in the time domain) then a lightning pulse excitation. The simulation geometry is illustrated in Fig. 7 for a sinusoidal input. The size of the simulation domain in the $\mathrm{x}$-direction is $0.4 \mathrm{~m}$ with periodic boundary conditions. The ground plane ends at $y=0$ and the top plate of the parallel plate waveguide begins at $y=0.8 \mathrm{~m}$. Two transparent E-field wave sources are placed in the z-direction. Source 1 is placed at $\mathrm{z}=-0.296 \mathrm{~m}$ and source 2 is placed at $0.296 \mathrm{~m}$. Two cells behind each wave source is a $0.02 \mathrm{~m}$ thick PML. Using two wave sources allows one to simulate an arbitrary reflection from the end of the parallel plate waveguide without having to model the actual load. In all of our FDTD simulations we will simulate a short circuit at source 2 by picking the amplitude of source 2 to be the negative of that of source 1 and delaying the pulse by $2 * 0.296 / \mathrm{c}$, where $\mathrm{c}$ is the speed of light. The inductive electric field that is produced is non-uniform, as shown in Fig. 7. The B-field is uniform over the antenna and is increased by a factor of two due to the short circuit condition. 


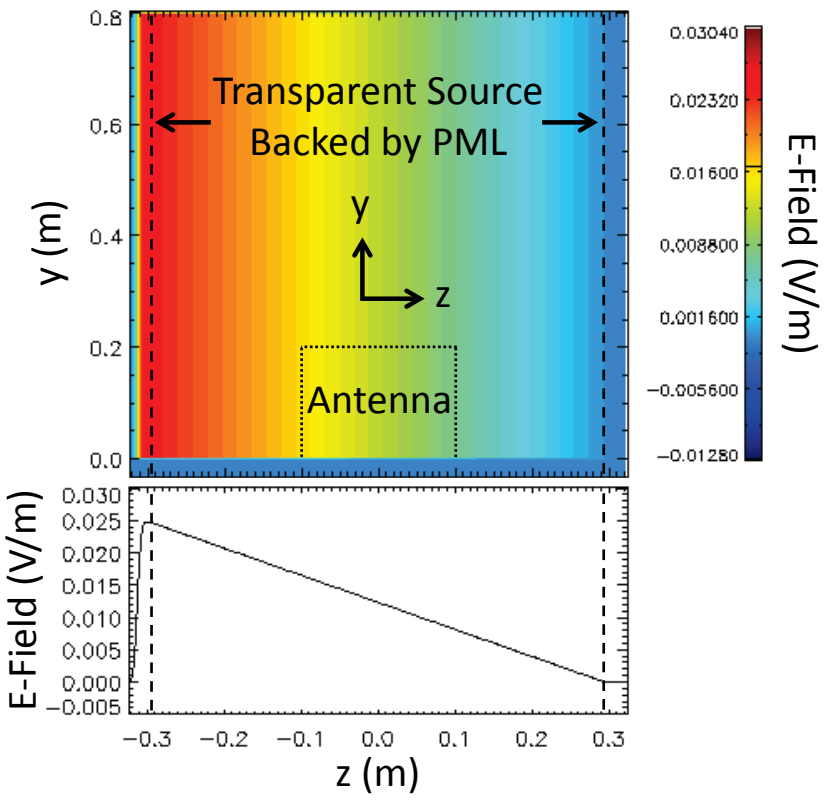

Fig. 7. Illustration of parallel plate geometry for FDTD simulations showing the inductive E-field near its peak value for a $1 \mathrm{~V} / \mathrm{m}, 1 \mathrm{MHz}$ sinusoidal input. The dotted line represents the location of the antenna (not simulated).

For the first several examples the input at source 1 will be $\left(1-\exp \left(-\mathrm{t}^{3} / 4 \mathrm{E}-22\right)\right) \cos (2 \pi \mathrm{ft}+0.25 \pi) \mathrm{V} / \mathrm{m}$, with $\mathrm{f}=1 \mathrm{MHz}$. If one were to simply use a sine or cosine the discontinuities in the function or its derivative introduces high frequencies into the simulation, causing the antenna to resonate, violating our assumption about a low frequency excitation. The exponential factor multiplying the cosine dampens this high frequency. The resultant E-field and B-field in the waveguide with no antenna present are shown in Fig. 8 for a half cycle of the cosine after the exponential factor has decayed. The Efield is shown at the two vertical sections of the folded antennas as well as at the center. Because the B-field is uniform over the antenna it is shown only at the center location $(\mathrm{z}=0)$. Note that the peak total E-field has been lowered by $\sim 80$ at the center of the antenna relative to the incident pulse, while the peak total B-field has been doubled relative to the incident pulse. The dashed lines in Fig. 8 are from simple closed form calculations to predict the fields and show excellent agreement with the simulated values.

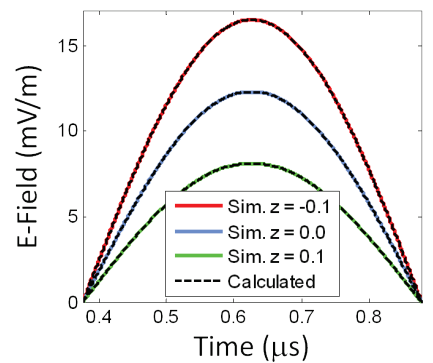

(a)

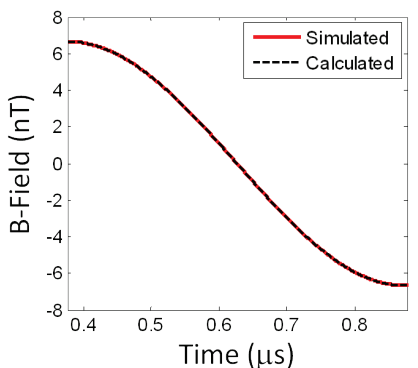

(b)
Fig. 8. The (a) E-field and (b) B-field in the empty cavity found from the FDTD simulation as well as calculated values from closed form expressions.
The first antenna simulations were performed for both the continuous and discontinuous folded antennas with $\mathrm{C}_{\mathrm{L} 2}$. The simulated and calculated voltages at the load $(z=-0.1 \mathrm{~m})$ and the parasitic gap $(\mathrm{z}=0.1 \mathrm{~m}$ for continuous case and $\mathrm{z}=0$ for discontinuous case) are shown in Figs. 9 and 10 . The calculated values were obtained using the E-field and B-field values at $\mathrm{z}=0 \mathrm{~m}$. Figs. 9 (a) and 10 (a) show the calculated voltage components due to the E-field coupling and the Bfield coupling. The voltages due to the E-field coupling have the same magnitude and polarity for the load and parasitic gap. The voltages developed for the B-field coupling are $180^{\circ}$ out of phase and have different magnitudes for load and parasitic gap. We also see that because the ratio of $\mathrm{E} / \mathrm{B}$ has changed, the voltages due to the B-field coupling are on the same order of magnitude as the E-field coupling and cannot be neglected. Figures 9 (b) and 10 (b) shows the results of the voltage from the simulation, as well as the calculated values found by summing the contributions from the E-field and B-field coupling $\left(\mathrm{v}_{\mathrm{E}}+\mathrm{v}_{\mathrm{B}}\right)$ for both the load and parasitic gap. We see that excellent agreement is obtained between the calculated and simulated values.

The last simulation with a sinusoidal excitation was for the continuous folded antenna with $\mathrm{C}_{\mathrm{L} 1}$. For this case the voltages developed at the load and parasitic gap for the B-field coupling are $\sim$ equal in magnitude but $180^{\circ}$ out of phase. The results are shown in Fig. 11. Figure 11 (a) shows the contributions to the voltages for E-field and B-field coupling.

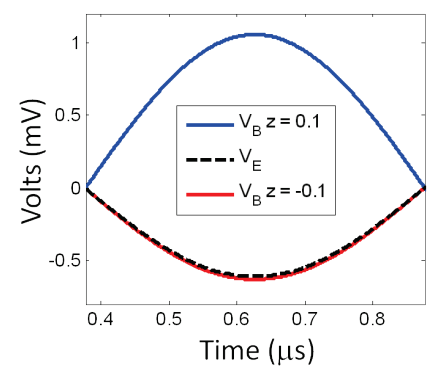

(a)

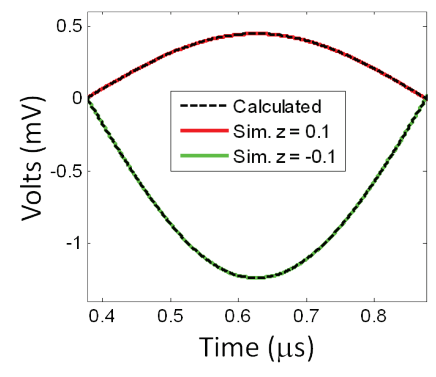

(b)
Fig. 9. For the continuous folded antenna with $\mathrm{C}_{\mathrm{L} 2}$ attached (a) the contributions to the voltage due to E-field and B-field coupling at the load and parasitic gap, and (b) the simulated voltages and calculated total voltages at the load and parasitic gap.

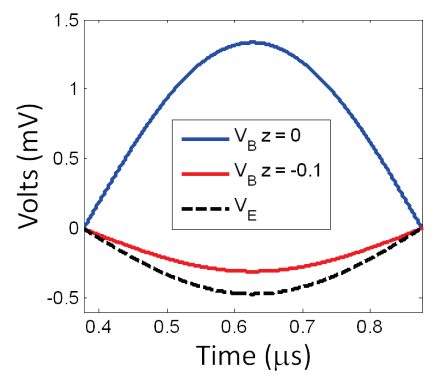

(a)

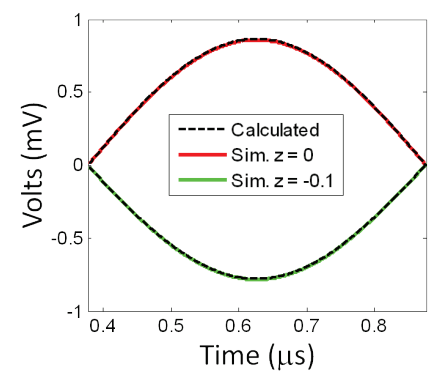

(b)
Fig. 10. For the discontinuous folded antenna with $C_{\mathrm{L} 2}$ attached (a) the contributions to the voltage due to E-field and B-field coupling at the load and parasitic gap, and (b) the simulated voltages and calculated total voltages at the load and parasitic gap. 
We see that the values are nearly equal in magnitude for the E-field and B-field coupling. The simulated and calculated total voltages at the load and parasitic gap are shown in Fig. 11 (b). We can see that the calculated and simulated values have excellent agreement for the load side. An expanded view of the parasitic gap voltage is shown in Fig. 11 (c). We see that the simulated and calculated total voltages don't agree as well and results in a percent difference of $\sim 17 \%$ at the peak The values of $C_{A B}$ and $C_{P B}$ were modified to $3.4525 \mathrm{pF}(1.2 \%$ change $)$ and $6.1475 \mathrm{pF}(0.7 \%$ change $)$, resulting in the corrected calculated value shown in the figure. This extremely sensitive case further validates our model as the small change in capacitance needed to obtain the excellent agreement between simulated and calculated voltages can easily be explained by small computational errors and the effect of the fringe capacitances introduced by removing the load from the antenna in the simulations to calculate $\mathrm{v}_{\mathrm{SE}}, \mathrm{C}_{\mathrm{AE}}$, and $\mathrm{C}_{\mathrm{L}}$.

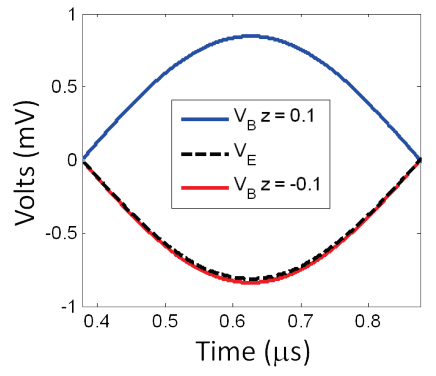

(a)

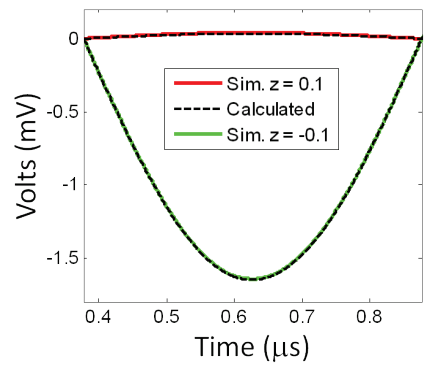

(b)

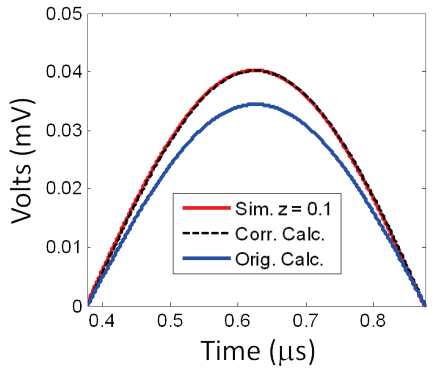

(c)
Fig. 11. For the continuous folded antenna with $\mathrm{C}_{\mathrm{L} 1}$ attached (a) the contributions to the voltage due to E-field and B-field coupling at the load and parasitic gap, (b) the simulated voltages and calculated total voltages at the load and parasitic gap, and (c) expanded view of the simulated and calculated total voltages at the parasitic gap using the original and corrected values for $\mathrm{C}_{\mathrm{AB}}$ and $\mathrm{C}_{\mathrm{PB}}$.

As discussed in the introduction, an important application for the coupling model being analysed is for when lightning strikes a facility. In order to validate that the coupling model developed is applicable to the entire frequency spectrum for lightning, a realistic lightning pulse is needed to excite the folded antenna structure. We will use a modified version of the Heidler lightning pulse discussed in [14] for the negative first stroke current.

The pulse has been rescaled such that the peak lightning $\mathrm{di} / \mathrm{dt}$ is $400 \mathrm{kA} / \mu \mathrm{s}$. It has been modified by shifting the pulse forward $2.5 \mu \mathrm{s}$ in order to shorten the computational resources needed to run the FDTD simulation. The pulse was then multiplied by $\left(1-\exp \left(-\mathrm{t}^{2} / 8 \mathrm{E}-13\right)\right)$ in order to remove discontinuities introduced by the time shift in the lightning current and its derivative. The exponential factor multiplying the shifted pulse decayed to one before the current and its derivative became appreciable. The resulting pulse is shown in Fig. 12. Also shown is a double exponential that has approximately the same peak di/dt. The double exponential model is not suitable for simulations due to the large discontinuity in $\mathrm{di} / \mathrm{dt}$ at $\mathrm{t}=0$. Figure 12 (d) displays the amplitude of the frequency spectrum for both the lightning pulse and its derivative, showing that they are both low frequency. For our validation simulations the lightning pulse is simply used as the E-field wave source. Figure 13 shows the resultant E-field at the two vertical sections of the folded antennas and the center, as well as the B-field at the center when empty cavity is excited by the Heidler lightning pulse.

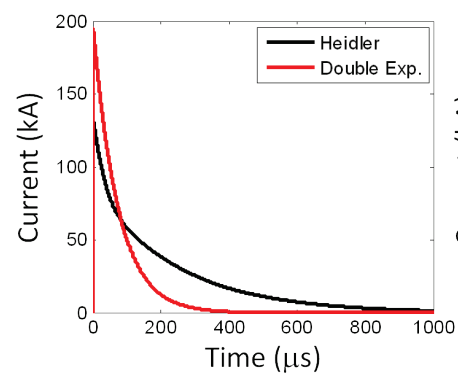

(a)

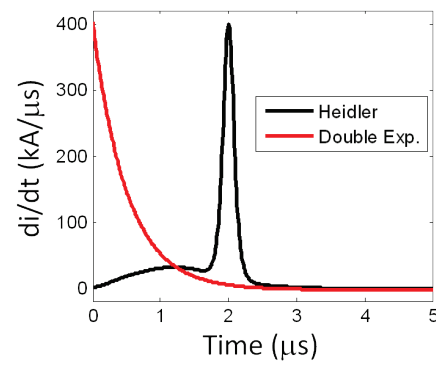

(c)

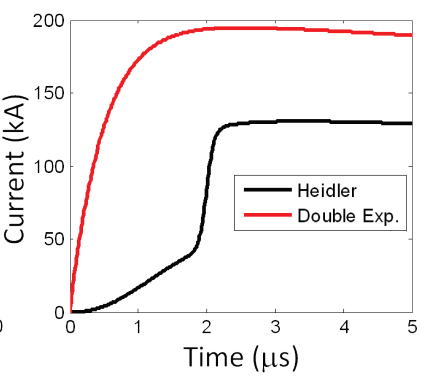

(b)

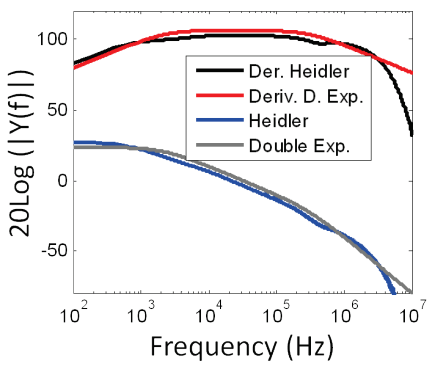

(d)
Fig. 12. For a Heidler and double exponential lightning pulse (a) the current, (b) an expanded view of the current near the peak di/dt, (c) the derivative, and (d) the frequency spectrum of the current and its derivative.

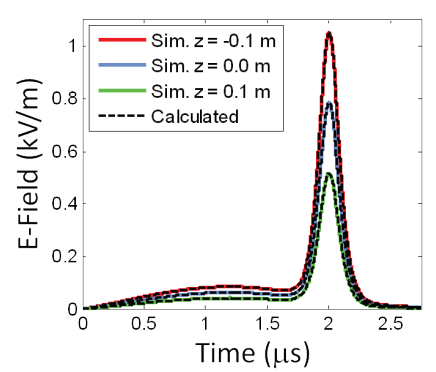

(a)

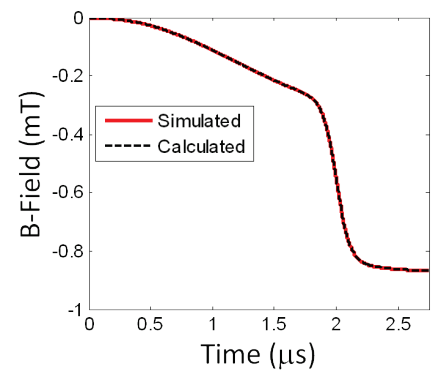

(b)
Fig. 13. The (a) E-field and (b) B-field in the empty cavity found from the FDTD simulation as well as calculated values from closed form expressions for the Heidler lightning pulse excitation. 
Finally, simulations were performed for both the continuous and discontinuous folded antennas with $\mathrm{C}_{\mathrm{L} 2}$ attached and a Heidler lightning pulse excitation. The simulated and calculated voltages at the load and the parasitic gap are shown in Figs. 14 and $15 . \quad$ We see that excellent agreement is obtained between the calculated and simulated values for both cases. This validates that our model is applicable to both E-field and B-field coupling for a realistic low frequency lightning pulse.

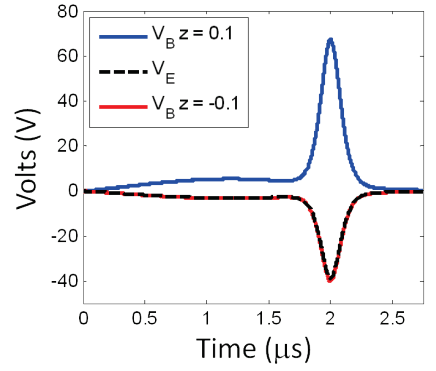

(a)

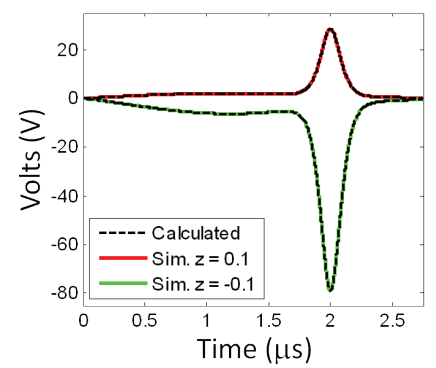

(b)
Fig.14. For the continuous folded antenna with $\mathrm{C}_{\mathrm{L} 2}$ attached and a Heidler lightning pulse excitation (a) the contributions to the voltage due to E-field and B-field coupling at the load and parasitic gap, and (b) the simulated voltages and calculated total voltages at the load and parasitic gap.

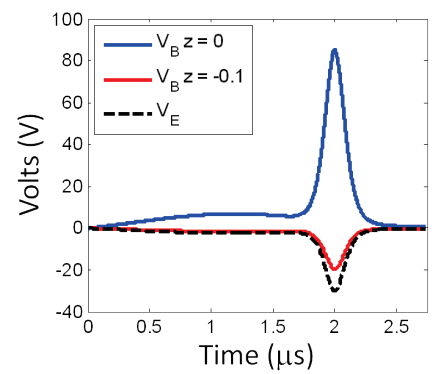

(a)

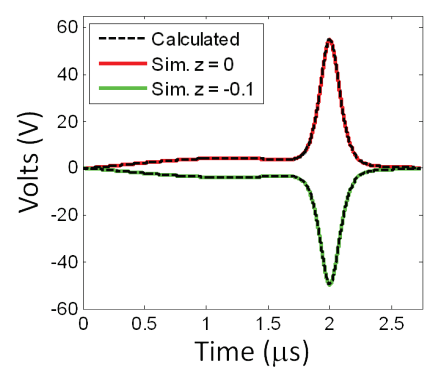

(b)
Fig.15. For the discontinuous folded antenna with $C_{\mathrm{L} 2}$ attached and a Heidler lightning pulse excitation (a) the contributions to the voltage due to E-field and B-field coupling at the load and parasitic gap, and (b) the simulated voltages and calculated total voltages at the load and parasitic gap.

\section{ACKNOWLEDGMENT}

The authors would like to thank Constantine A. Hrousis of LLNL for providing funds for lightning effects research, and Carol S. Richardson of LLNL for administrative support.

This work performed under the auspices of the U.S. Department of Energy by Lawrence Livermore National Laboratory under Contract DE-AC52-07NA27344.

\section{REFERENCES}

[1] M. P. Perkins, M. M. Ong, C. G. Brown Jr., and R. D. Speer, "Analysis of Conductor Impedances Accounting for Skin Effect and Nonlinear Permeability," in Proc. of the $18^{\text {th }}$ Int. Pulsed Power Conf., 2011.

[2] M. P. Perkins, M. M. Ong, E. W. Crull, and C. G. Brown Jr., "Modeling Techniques Used to Analyze Safety of Payloads for Generic Missile Type Weapons Systems During an Indirect Lightning Strike," in Proc. of the $31^{\text {st }}$ Annual Ant. Meas. Tech. Asso. Symp., 2009.

[3] M. P. Perkins, M. M. Ong, R. D. Speer, and C. G. Brown Jr., "Analysis of a Small Loop Antenna with Inductive Coupling to Nearby Loops," IEEE Trans. Electromagn. Compat., vol. 53, no. 4, pp. 900-908, Nov. 2011.

[4] R. King, "The Rectangular Loop Antenna as a Dipole," IRE Trans. Ant. Prop., vol. 7, no. 1, pp. 53-61, Jan. 1959.

[5] C. W. Harrison Jr., and R. W. P. King, "Folded Dipoles and Loops," IRE Trans. Ant. Prop., vol. 9, no. 2, pp. 171-187, Mar. 1961.

[6] D. H. Kwon, and Y. Kim, "Small Low-Profile Loop Wideband Antennas with Unidirectional Radiation Characteristics," IEEE Ant. Prop., vol. 55, no. 1, pp. 72-77, Jan. 2007.

[7] B. Josephson, B, "The Quarter-Wave Dipole," in Proc. IRE Wescon Conv., 1957, p. 77

[8] M. Kanda, "An Electromagnetic Near-Field Sensor for Simultaneous Electric and Magnetic-Field Measurements," IEEE Trans. Electromagn. Compat., vol. 26, no. 3, pp. 102-110, Aug. 1984.

[9] M. E. G. Upton, and A. C. Marvin, "Improvements to an Electromagnetic Near-Field Sensor for Simultaneous Electric and Magnetic Field Measurements," IEEE Trans. Electromagn. Compat., vol. 35, no. 9, pp. 96-98, Feb. 1993.

[10] K. P. Esselle, and S. S. Stuchly, "Resistively Loaded Loop as a PulseReceiving Antenna," IEEE Trans. Ant. Prop., vol. 38, no. 7, pp. 11231126, July 1990.

[11] M. J. Underhill, and M. Harper, "Simple Circuit Model of Small Tuned Loop Antenna Including Observable Environmental Effects," Elect. Lett., vol. 38, no. 18, pp. 1006-1008, Aug. 2002.

[12] M. J. Underhill and M. J. Blewett, "Unidirectional Tuned Loop Antennas Using Combined Loop and Dipole Modes," in Proc. $8^{\text {th }}$ Inter. Conf. HF Radio Systems and Tech., 2000, p. 37.

[13] H. Whiteside, and R. W. P. King, "The Loop Antenna as a Probe," IEEE Trans. Ant. Prop., vol. 12, no. 3, pp. 291-297, May 1964.

[14] W. R. Gamerota, J. O. Elismé, M. A. Uman, and V. A. Rakov, "Current Waveforms for Lightning Simulation," IEEE Trans. Electromagn. Compat., Accepted for Publication, 2012. 